\title{
Gulf Cooperation Council Clinical Trials in the Pursuit of Medications for COVID-19
}

\author{
Bassam Ali JABER ${ }^{\mathrm{a}}$, Mowafa HOUSEH ${ }^{\mathrm{a}}$, Zubair SHAH ${ }^{\mathrm{a}}$, M.Sohel RAHMAN ${ }^{\mathrm{c}}$, \\ Mounir HAMDI ${ }^{\mathrm{a}}$, Kabir H BISWAS ${ }^{\mathrm{b}}$ and Tanvir ALAM ${ }^{\mathrm{a}, 1}$ \\ ${ }^{a}$ College of Science and Engineering, Hamad Bin Khalifa University, Doha, Qatar \\ ${ }^{\mathrm{b}}$ College of Health and Life Sciences, Hamad Bin Khalifa University, Doha, Qatar \\ ${ }^{\mathrm{c}}$ Department of Computer Science \& Engineering, Bangladesh University of \\ Engineering and Technology, Dhaka, Bangladesh
}

\begin{abstract}
Tremendous changes have been witnessed in the post-COVID-19 world. Global efforts were initiated to reach a successful treatment for this emerging disease. These efforts have focused on developing vaccinations and/or finding therapeutic agents that can be used to combat the virus or reduce its accompanying symptoms. Gulf Cooperation Council (GCC) countries have initiated efforts on many clinical trials to address the efficacy and the safety of several therapeutic agents used for COVID-19 treatment. In this article, we provide an overview of the GCC's clinical trials and associated drugs' discovery process in the pursuit of an effective medication for COVID-19.
\end{abstract}

Keywords. Coronavirus, COVID-19, Drug, GCC, Qatar.

\section{Introduction}

The fast and widespread COVID-19 pandemic compelled the medical society to use all resources to combat the virus as quickly as possible. One approach was to use certain medications already related to inhibiting virus replications, human cell invasions, or preventing inflammatory reactions that can lead to further deterioration or failure in numerous body organs [1]. In this article, we investigated the clinical trials that are currently under different phases of completion in the Gulf Cooperation Council (GCC) countries, which include Qatar, Kuwait, Oman, Saudi Arabia, UAE and Bahrain.

\section{Methods}

Detailed information on the clinical trials aimed towards finding therapeutic solutions for COVID-19 was collected from https://clinicaltrials.gov/ct2/covid_view (download date: May 07, 2021). Each clinical trial was then analyzed to understand the medications under investigation in the GCC countries.

1 Corresponding Author, Tanvir Alam, College of Science and Engineering, Hamad Bin Khalifa University, Doha, Qatar; E-mail: talam@hbku.edu.qa. 


\section{Results and Discussions}

In total, we found 27 clinical trials currently underway in GCC countries. Among them, 12, 8, and 5 clinical trials are being conducted in Saudi Arabia (KSA), Qatar, and Kuwait, respectively. Only one trial each was enrolled from the UAE and Bahrain. The medications investigated mainly fall into antiviral, anti-inflammatory, and anticoagulator categories. We also note additional drugs that have been repurposed as they exhibited some anti-viral, immune-modulating, or other effects. We also summarize the drugs in Table 1 and provide a brief of ongoing trials in GCC countries.

Table 1. List of drugs that are under clinical trials in GCC countries

\begin{tabular}{lll}
\hline \multicolumn{1}{c}{ Drug Family } & \multicolumn{1}{c}{ Drug name } & \multicolumn{1}{c}{ Clinical Trials } \\
\hline Anti-viral & Favipiravir, Remdesivir, Darunavir/ & NCT04387760, NCT04529499, \\
(Direct) & Lopinavir & NCT04464408, NCT04302766, \\
& & NCT04425382 \\
Anti-viral & Hydroxychloroquine, Artemisinin/ & NCT04437693, NCT04349592, \\
(Repurposed) & Artesunate, ACE Inhibitors and Angiotensin & NCT04394442, NCT04387240, \\
& II Receptor Blockers & NCT04357535 \\
Anti- & Pioglitazone, Anakinra, Zafirlukast, & NCT04604223, NCT04643678, \\
inflammatory & Tocilizumab/Siltuximab, Omega 3 Oil & NCT04486521, NCT04553705, \\
& & NCT04836052 \\
Anti-coagulant & Bivalirudin & NCT04445935 \\
Miscellaneous & Iloprost, Oestrogen, Alpha One Antitrypsin, & NCT04445246, NCT04853069, \\
& Colchicine, Interferon-Beta, Aspirin, & NCT04385836, NCT04324463, \\
& Rivaroxaban & NCT04468139 \\
\hline
\end{tabular}

\subsection{Antiviral Drugs}

Favipiravir: This antiviral is being used to treat unresponsive or re-emerging viral influenza strains in contrast to conventional anti-viral treatment [2]. Favipiravir

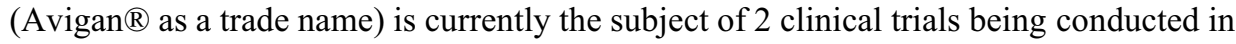
Kuwait and KSA (NCT04529499 and NCT04464408, respectively). While both clinical trials are evaluating the efficacy and safety of Favipiravir in COVID-19 treatment, they differ in the outcome measurements. The first one explores "Time to resolution of hypoxia" as a primary outcome while the other one is being conducted on "Time from randomization to negativity in the RT-PCR nucleic acid test for COVID-19 within 15 days of randomization".

Remdesivir: Remdesivir is similar to Favipiravir as it also selectively inhibits RNAdependent RNA polymerase (RdRp) [3]. The Kuwait-based trial (NCT04302766) has not determined the outcome measures of the interventions being applied throughout the clinical trial.

Darunavir/Lopinavir: Both of these antivirals are HIV protease inhibitors employed in COVID-19 treatment due to their ability to prevent further infection of the virus. The clinical trial (NCT04425382) taking place in Qatar aims to compare the efficacy and safety outcomes of Darunavir/Cobicistat versus Lopinavir/Ritonavir in treating pneumonia associated with COVID-19.

\subsection{Antiviral Drugs (Repurposed)}

Hydroxychloroquine (HCQ): This anti-malarial drug, which is also used in rheumatoid arthritis and other autoimmune diseases, blocks viral entry into the cells through endosomal acidification [4]. In Bahrain, clinical trial NCT04387760 is a pilot study 
exploring the ability to create a comparison between HCQ, Favipiravir, and the routine care using the time frame until hospital discharge or death as the measure of the primary outcome. HCQ was also evaluated for its safety, efficacy, and effectiveness in Post Exposure Prophylaxis (PEP) for health care providers in Qatar (NCT04437693). Another clinical trial in Qatar, NCT04349592, addressed the value of adding Azithromycin, an antibiotic in the Macrolide family that exhibits anti-inflammatory effects, to HCQ in COVID-19 treatment.

Artemisinin/Artesunate: An antimalarial combination that shows antiviral activity. It also has an anti-inflammatory effect, which is attributed to its ability to reduce the level of inflammatory cytokines. The reduction avoids both the cytokine storm as well as inflammatory organ injuries for high-risk patients [5]. A clinical trial taking place in KSA is evaluating the efficacy of this combination.

Angiotensin-Converting Enzyme Inhibitors (ACE-I) and Angiotensin II Receptor (ARB) Blockers: The role of ACE and ARB in COVID-19 prognosis is still controversial. The hypothesis suggests that the SARS-CoV-2 uses ACE2 protein expressed on alveolar cell surfaces to enter the cell. Accordingly, patients who are hypertensive and receiving ACEs or ARBs are at high risk for potentially developing Acute Respiratory Distress Syndrome (ARDS). Another hypothesis denies these claims as they are unsupported by virus binding. Additionally, internalization may not require the catalytic activity of the protein [6]. These inhibitors may cause alteration in the levels of the protein on the cell membrane, which can affect virus binding. Additionally, a clinical trial is taking place in KSA and targeting only hypertensive patients who are on $\mathrm{ACE}$ or ARB to assess the impact of ARB and ACE-I on a COVID-19 prognosis. The primary outcome would be the time frame from the date of enrollment until discharge from hospital or death; whichever comes first.

\subsection{Anti-Inflammatory Drugs}

Pioglitazone: Researchers have claimed that this insulin sensitizing, anti-diabetic agent reduces chronic inflammation in T2DM patients. Moreover, it enhances the plasma level of anti-inflammatory agents. This feature has been used to improve the clinical outcome (NCT04604223) of the COVID-19 disease in T2DM patients.

Anakinra: An interleukin (IL)-1 receptor antagonist used in many inflammatory diseases such as rheumatoid arthritis, familial Mediterranean fever. In the clinical trial NCT04643678, it is used to avoid Cytokine storm syndrome, thus reducing the time for mechanical ventilation and improving the clinical outcome of patients with moderate to severe COVID-19 symptoms [7].

Zafirlukast: A leukotriene receptor antagonist used for treating chronic asthma. Its efficacy is being investigated in a clinical trial in KSA (NCT04871828). The primary investigation on this trial is time frame of clinical improvement.

Tocilizumab/Siltuximab: Interleukin-6 Antagonists (Anti IL6) have been used as antirheumatic. Tocilizumab and Siltuximab Inhibition of IL-6 of receptors may lead to the reduction of cytokine and acute phase reactant production [8]. The efficacy of such an approach is being investigated in a KSA-based clinical trial, NCT0448652, which explores a primary outcome of ventilator-free days.

Omega 3 Oil: Under a clinical trial (NCT04836052) in Qatar, Omega-3-oil has been shown to have fewer proinflammatory mediators that may have immunomodulating, anti-inflammatory and antiviral effects. These features can be employed to improve the clinical outcomes of patients admitted to ICU due to COVID-19. 


\subsection{Anticoagulation Drugs}

Bivalirudin: In the ARDS associated with COVID-19 infection is contributed to the activation of the coagulation system. Administrating Bivalirudin, an anticoagulant with a direct thrombin inhibition effect, can have positive effects in improving a patient's respiratory status. An ongoing clinical trial (NCT04445935) in Qatar is investigating the effects of Bivalirudin.

\subsection{Miscellaneous}

Hydrogen Peroxide, Povidone Iodine, Cetylpyridinium Chloride: Sodium Hypochlorite $(0.1 \%$ solution $)$ is an oral mouthwash used as a local antiseptic in dental procedures to clear saliva's viral load in COVID-19 patients. A clinical trial is being conducted to test the efficacy of this combination in KSA, with the change in viral load as the primary outcome. Additionally, these local antiseptics are being used to prevent the transmission of the virus rather than treatment. Miscellaneous drugs such as Iloprost, Oestrogen, Alpha One Antitrypsin, Colchicine, Interferon-Beta, Aspirin, and Rivaroxaban, etc., as well as plasma therapy (NCT04474340) are also in clinical trials utilized for exploring treatment plans of COVID-19. We considered clinical trials registered till May 2021 and this is a limitation of this study. The full list of the clinical trials can be found at: https://github.com/tanviralambd/GCC_ClinicalTrials.

\section{Conclusions}

GCC countries are working to curb the spread of COVID-19 and find a therapeutic solution. While the vaccination efforts are continuing in the GCC countries at a reasonable speed, no particular drug exists that may be considered a solution for this disease. A major stream of the drugs in clinical trials are antivirals (known or repurposed) that can combat the virus with acceptable levels of patient safety. Moreover, antiinflammatory drugs are also under trial to keep patients free from inflammation, leading to ARDS, cytokine storm, and other consequences. Ideally, these clinical trials conducted in the GCC will provide more insight into the efficacy of drugs against COVID-19.

\section{References}

[1] Khan JY, et al. Toward Preparing a Knowledge Base to Explore Potential Drugs and Biomedical Entities Related to COVID-19: Automated Computational Approach. JMIR Med Inform. 2020;8:e21648.

[2] Agrawal U, Raju R, Udwadia ZF. Favipiravir: A new and emerging antiviral option in COVID-19. Armed Forces Med J India. 2020;76:370-6.

[3] Rochwerg B, et al. Remdesivir for severe covid-19: a clinical practice guideline. BMJ. 2020;370:m2924.

[4] Schaper C. A Mechanism of Action for Hydroxychloroquine and Azithromycin to Inhibit Coronavirus Disease COVID-19 [Internet]. Available from: http://dx.doi.org/10.26434/chemrxiv.12148914.v1.

[5] Uzun T, Toptas O. Artesunate: could be an alternative drug to chloroquine in COVID-19 treatment? Chin Med. 2020;15:54.

[6] Javanmard SH, Heshmat-Ghahdarijani K, Vaseghi G. Angiotensin-converting-enzyme inhibitors (ACE inhibitors) and angiotensin II receptor blocker (ARB) use in COVID-19 prevention or treatment: A paradox [Internet]. Infection Control \& Hospital Epidemiology. 2021. p. 118-9. 
[7] Navarro-Millán I, Sattui SE, Lakhanpal A, Zisa D, Siegel CH, Crow MK. Use of Anakinra to Prevent Mechanical Ventilation in Severe COVID-19: A Case Series [Internet]. Arthritis \& Rheumatology. 2020. p. 1990-7. Available from: http://dx.doi.org/10.1002/art.41422

[8] Khan FA, Stewart I, Fabbri L, Moss S, Robinson K, Smyth AR, et al. Systematic review and metaanalysis of anakinra, sarilumab, siltuximab and tocilizumab for COVID-19. Thorax. 2021;76:907-19. 